\title{
AN UNUSUAL CASE OF FAHR'S DISEASE
}

\author{
George de Albuquerque Cavalcanti-Mendes', Gervásio Teles C. de Carvalho², \\ Paulo Pereira Christo 3 , Leandro F. Malloy-Diniz ${ }^{4}$, Atos Alves de Sousa ${ }^{2}$
}

Fahr's disease (FD), also called idiopathic striopallidodentate calcinosis, nonarteriosclerotic cerebral calcification or idiopathic basal ganglia calcification ${ }^{1,2}$, is a rare clinical entity characterized by movement disorders, dementia and behavioral disorders related to symmetric and bilateral calcifications of the basal ganglia. Sporadic and family cases have been reported ${ }^{1-3}$. FD should be differentiated from Fahr's syndrome. The latter is defined as symmetric and bilateral calcifications of the basal ganglia associated with neuropsychiatric manifestations that preferentially occur in patients with parathyroid disorders, especially hypoparathyroidism².

We report the case of a patient who presented dementia and a stroke-like episode in the absence of extrapyramidal alterations or disturbances in calcium and phosphorus metabolism. Cranial computed tomography (CT) revealed extensive diffuse calcifications located in unusual areas.

\section{CASE}

A 51-year-old unmarried Caucasian man, a topographer's assistant with eight years of schooling, sought our Service in 1993 with symptoms of odynophagia after manipulation of dyes at home. The patient had a history of delayed neuropsychomotor development, especially learning difficulties in school. Examination revealed a cognitive decline, mainly on impairment of recent memory, affective alterations as apathy, anhedonia, emotional lability, excessive fear, without compromise of the cranial nerves, eye's fundus, muscle strength, myotactic reflexes, gait, coordination and balance, and without extrapyramidal sign. The diagnosis of depressive disorder was made and the patient was treated with fluoxetine.

Neuropsychological evaluation showed an overall intelligence below the average expected for his age and educational level. Skills related to the understanding of language and nomination (which involved semantic integrity) were at the lowest normal limit. In addition, important deficits related to executive functions (particularly planning skills, inhibitory control, cognitive flexibility and categorization), attention, auditory-verbal, learning and recognition memories, susceptibility to the effect of proactive and retroactive interference, and an increased forgetfulness rate were observed.

Laboratory tests including erythrocyte sedimentation rate, C-reactive protein, ANF, VDRL, ceruloplasmin, serum copper, CPK, lactate, calcium ion, phosphorus, magnesium, urinary calcium, hepatic and renal function tests, protein electrophoresis, TSH, free T4, and parathormone were all normal. The EEG showed no alterations. Cranial CT scans (Fig 1) revealed bilateral hyperdense images located at the level of the caudate nucleus, putamen, globus pallidus, frontal and temporal subcortical white matter, semioval centers and cerebellum. FD was then diagnosed.

Thirteen years after the diagnosis, the patient load a sudden right hemiparesis associated with dysarthria. A CT scan revealed no new alterations. Brain magnetic ressonance imaging (MRI) showed signal alterations as a result of calcifications in both brain hemispheres, dentate nuclei, basal ganglia and subcortical white matter, and cortical hypotrophy in the absence of ischemic signs (Fig 2). An informed consent was given by the patient for this publication.

\section{DISCUSSION}

FD is characterized by clinical heterogeneity and can be asymptomatic (more frequent among middle-aged patients) or associated with neuropsychiatric manifestations ${ }^{1-5}$. The true prevalence of FD is unknown, but an incidence of basal ganglia calcifications ranging from $0.24 \%$ to $2 \%$ has been reported in consecutive radiologic studies, showing an evident relationship with increasing age $\mathrm{e}^{4,6-8}$. Tedrus et al. ${ }^{4}$ reported an incidence of $0.68 \%$ among 3.662 cranial CT scans analyzed.

It should be emphasized that there are other conditions that can also produce intracerebral calcifications. These conditions include endocrinopathies (hypothyroidism, hypogonadotrophic hypogonadism), systemic diseases (systemic scleroderma, systemic lupus erythematosus), infections (toxoplasmosis, neurocysticercosis, German measles, neurobrucellosis, HIV), primitive or secondary calcified brain tumors, and various diseases such

\section{UM CASO ATÍPICO DE DOENÇA DE FAHR}

${ }^{1}$ Resident, Department of Neurosurgery, Santa Casa de Belo Horizonte, Belo Horizonte MG, Brazil; ${ }^{2}$ Assistant, Department of Neurosurgery, Santa Casa de Belo Horizonte and Faculdade de Ciências Médicas de Minas Gerais, Belo Horizonte MG, Brazil; ${ }^{3}$ Assistant, Department of Neurology, Santa Casa de Belo Horizonte, Belo Horizonte MG, Brazil; ${ }^{4}$ Assistent I, Department of Psycology, Universidade Federal de Minas Gerais, Belo Horizonte MG, Brazil. 

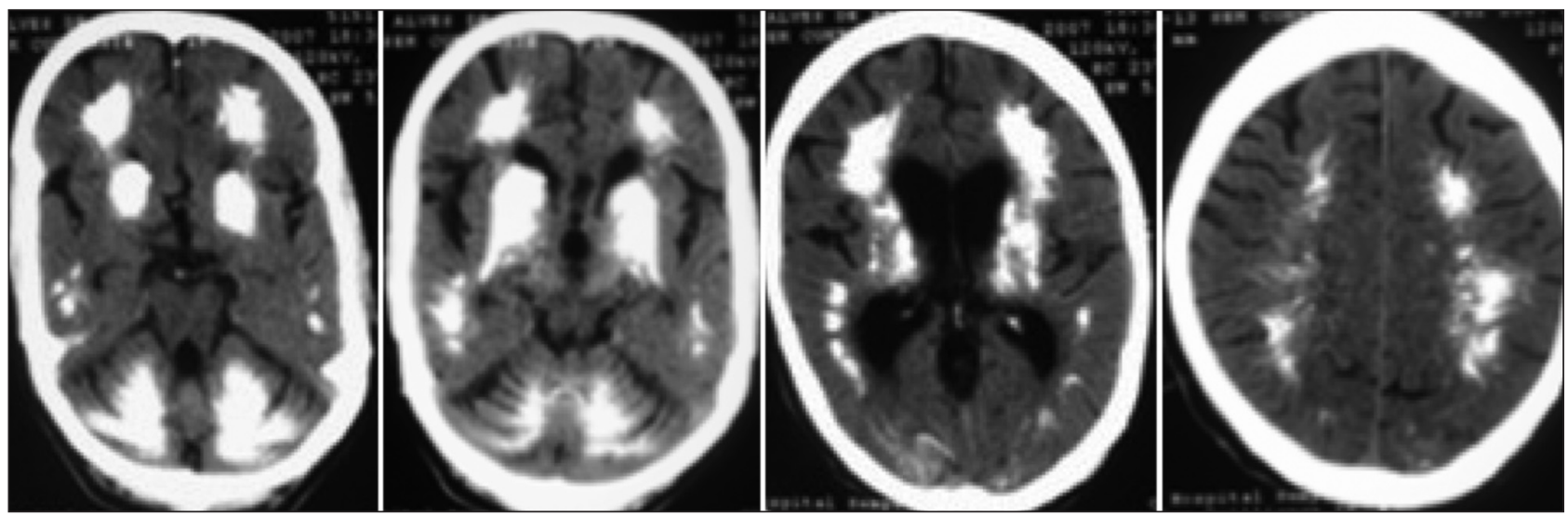

Fig 1. Cranial CT scans showing calcifications in the basal ganglia, thalamus, frontal, temporal and occipital periventricular white matter, semioval centers, and cerebellum.
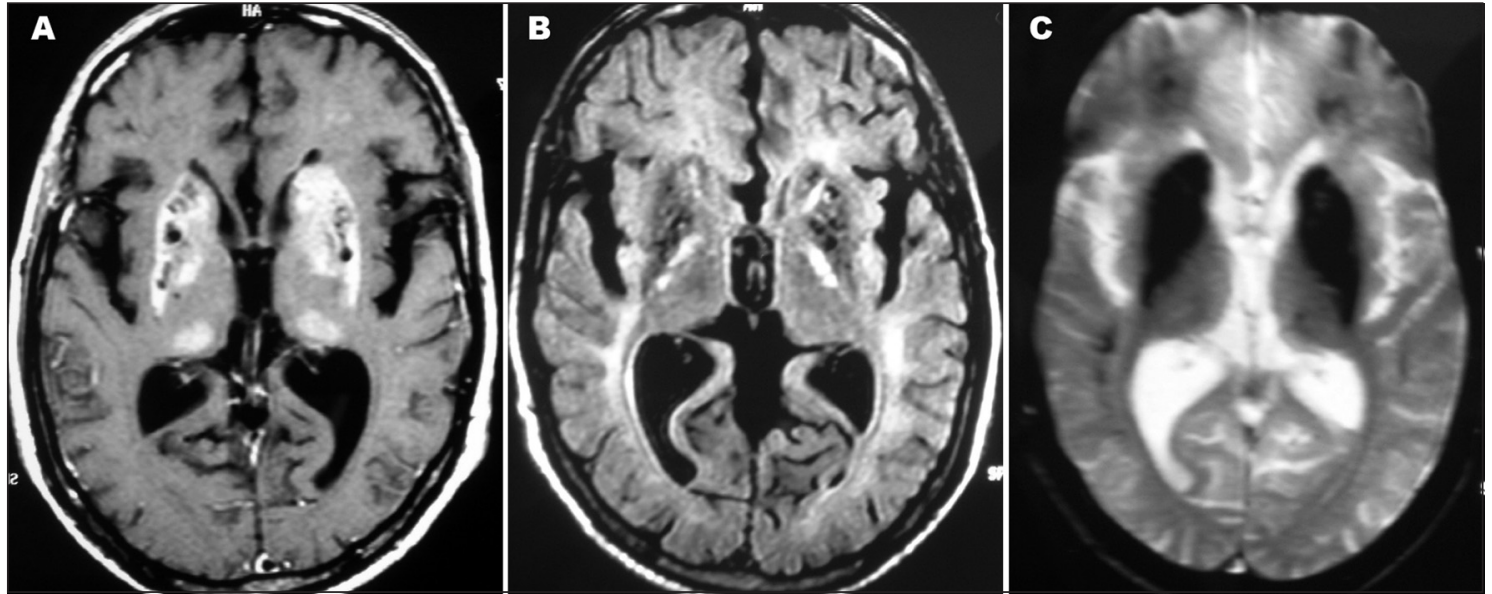

Fig 2. Axial MRI showing alterations in basal ganglia: [A] hyperintense signal on a T7-weighted sequence without enhancement after gadolinium injection; $[B]$ heterogenous signal on a FLAIR image with clearly visible bilateral frontotemporal volume reduction; [C] strongly hypointense signal on a T2-weighted gradient-echo image.

as tuberous sclerosis, mitochondrial encephalopathy, myotonic muscle dystrophy, measles and smallpox encephalitis, post-anoxia disorders, phacomatosis, Cockayne syndrome, neonatal anoxia, idiopathic hemochromatosis, heavy metal and carbon monoxide intoxication, treatment with methotrexate, and radiotherapy ${ }^{2,4,7,9}$. These diseases cause bilateral and non-symmetric cerebral calcifications mainly located in the basal ganglia and cerebellum ${ }^{2,4}$.

FD should be diagnosed based on clinical aspects, neuroimaging findings and the exclusion of other primary causes. It may occur in a sporadic or familial manner ${ }^{2,3,5}$. Geschwind et al. ${ }^{10}$, in a genetic study, described a dominant autosomal inheritance of the hereditary form of FD and suggested that the disease is caused by mutations in genes located on the long arm of chromosome 14.

The physiopathological mechanism of FD is not completely understood. Anatomopathological alterations mainly were observed in small vessels, perivascular regions, neuroglia and neurons ${ }^{2,5}$. Using electron microsco- py, Kobayashi et al." proposed, as a physiopathological mechanism, initial damage to pericytes, promoting the accumulation of mucopolysaccharides in their cytoplasm, followed by mineral deposits. Hagiwara et al..$^{12}$ suggested abnormalities in intracranial oxygen metabolism were related to the physiopathology of FD after the observation of elevated cerebrospinal fluid lactate levels in a typical case of the disease.

The main neurological manifestations of FD include motor disorders such as parkinsonism, dystonia, tics, speech alterations, epilepsia, and motor deficits, which are probably due to involvement of the frontostriatal motor system ${ }^{2,3,13}$. Weisman et al. ${ }^{13}$ reported a case of FD with motor deficits whose autopsy revealed wallerian degeneration of the corticospinal tract in addition to the typical calcifications.

The most frequent neuropsychiatric syndromes associated with FD are schizophrenia-like psychosis, mood and personality disorders, obsessive-compulsive symptoms, and 
cognitive disorders, notably executive dysfunctions. These conditions have been suggested to be caused by corticosubcortical disconnection mediated by the basal ganglia, especially involving the frontostriatal and limbic circuits ${ }^{1,7,8,13,14}$.

FD has been associated with impairment of various cognitive modalities. Cartier et al. ${ }^{5}$ evaluated the cognitive performance of three sisters with the disease, with all three presenting impairment of verbal and visual memory, planning, processing rate, attention, and visuoconstruction. Deficits in executive functions have been reported to be prominent and to occur early in FD, followed by memory difficulties and mood alterations ${ }^{12}$. Wing et al. ${ }^{8}$ described two cases of patients with personality and behavioral disorders, suggesting the involvement of frontal subcortical circuits as a hypothetical model for the cognitive and psychiatric problems observed in FD and emphasizing their relevance for the differential diagnosis of frontal syndromes. Benke et al. ${ }^{15}$ performed a PET scan on a patient with neuropsychiatric symptoms related to FD which demonstrated glucose hypometabolism in the basal ganglia and frontal lobes. The authors suggested that subcortical lesions may only present as psychiatric symptoms in the absence of any significant motor manifestations. Shouyama et al. ${ }^{16}$, using brain SPECT to evaluate regional blood flow in a patient with FD presenting schizophrenia-like psychosis, suggested that psychiatric symptoms related to the disease might be caused by disruption of cortico-subcortical neural circuits.

The most common radiologic feature of FD is the presence of small bilateral intracranial calcifications which are usually restricted to the globus pallidus, but may also affect the putamen, caudate nucleus, thalamus, dentate nucleus and white matter of the cerebral hemispheres ${ }^{3,14,16,17}$. Lester et al..$^{17}$ reported an atypical case of FD which developed stroke-like symptoms and progressed to extrapyramidal syndrome. A cranial CT scan showed diffuse calcifications in the cerebellar hemispheres, putamen and temporal periventricular and subcortical white matter, and also involved the tegmentum pontis and mesencephalic peduncle.

In the present case, the patient developed psychiatric disorders typical of FD in the absence of any laboratory alterations. However, the patient presented atypical manifestations such as early cognitive decline and brachiocrural paresis which, together with personality disorder, suggest the involvement of frontostriatal-thalamic-cortical dorsal and basal pathways. Cranial CT demonstrated extensive bilateral and symmetric intracranial calcifications located at usual sites such as the basal ganglia and cerebellum and at unusual sites such as the temporal and frontal periventricular white matter and semioval centers. Brain MRI confirmed the CT scan findings and showed no signs of pyramidal involvement.

This case strengthened that FD should be included in the differential diagnosis of patients who present psychiatric symptoms associated with motor disorders when calcifications are found mainly in the basal ganglia.

\section{REFERENCES}

1. Kummer A, Castro M, Carameli P, Cardoso F, Teixeira AL. Alterações comportamentais graves em paciente com doença de Fahr. Arq Neuropsiquiatr 2006;64:645-649.

2. Chevalier D, Tillon J. A cause of cerebral calcifications not to be ignored: Fahr syndrome. Rev Med Interne 2005;26:668-677.

3. Manyam BV. What is and what is not "Fahr's disease". Parkinsonism Relat Disorder 2005;11:73-80.

4. Tedrus GMAS, Fonseca LC, Nogueira EJ. Basal ganglia calcification on computed tomography: clinical characteristics in 25 patients. Arq Neuropsiquiatr 2006;64:645-649.

5. Cartier RL, Passig VC, Gormaz WA, López CJ. Cambios neuropsicológicos en la enfermidad de Fahr. Rev Méd Chile 2002; 130:1383-1390.

6. Fahr T. Idiopathische Verkalkung der Hirngefässe. Zentralblatt für allgemeine Pathologie und pathologische Anatomie 1930;50:129-133.

7. Shakibai SV, Johnson JP, Bourgeois JA. Paranoid delusions and cognitive impairment suggesting Fahr's disease. Psychosomatics 2005;46:569-572.

8. Lam JSP, Fong SYY, Yiu GC, Wing YK. Fahr's disease: a differential diagnosis of frontal lobe syndrome. Hong Kong Med J 2007;13:75-7.

9. Faria AV, Pereira IC, Nanni L. Computerized tomography findings in Fahr's syndrome. Arq Neuropsiquiatr 2004;62:789-792.

10. Geschwind DH, Loginov M, Stern JM. Identification of a locus on chromosome 14q for idiopathic basal ganglia calcification (Fahr's disease). Am J Hum Genet 1999;65:764-772.

11. Kobayashi S, Yamadori I, Ohmori M. Idiopathic nonatherosclerotic cerebral calcification (Fahr's disease): an electron microscopic study. Acta Neuropathol 1987;73:62-66.

12. Hagiwara N, Ooboshi H, Ishibashi M, et al. Elevated cerebrospinal fluid lactate levels and the pathomechanism of calcification in Fahr's disease. Eur J Neurol 2006;13:539-543.

13. Weissman DC, Yaari R, Hansen LA, Thal LJ. Density of the brain, decline of the mind (an atypical case of Fahr disease). Arch Neurol 2007;64:756.

14. Schimdt U, Mursch K, Halatsch ME. Symmetrical intracerebral and intracerebellar calcification ("Fahr's disease"). Funct Neurol 2005;20:15.

15. Benke T, Karner E, Seppi K, Delazer M, Marksteiner J, Donnemiller E. Subacute dementia and imaging correlates in a case of Fahr's disease. J Neurol Neurosurg Psychiatry 2004;75:1163-1165.

16. Shouyama M, Kitabata Y, Kaku T, Shinosaki. Evaluation of regional cerebral blood flow in Fahr's disease with schizophrenia-like psychosis: a case report. AJNR Am J Neuroradiol 2005; 26:2527-2529

17. Lester J, Zúniga C, Díaz S, Rugilo C. Micheli F. Diffuse intracranial calcinosis (Fahr's disease). Arch Neurol 2006;63:1806. 